\title{
Etiological Trends and Epidemiological Profile of Tropical Fever in Children Presenting with Acute Undifferentiated Fever at a Tertiary Care Centre in North India

\author{
Monika Matlani' ${ }^{1}$, Pramod Kumar ${ }^{2}$, Shyam S Meena ${ }^{2}$, Mohan Vashistha ${ }^{1}$ and Vinita \\ Dogra 1
}

${ }^{1}$ Department of Microbiology, Vardhman Mahavir Medical College and Safdarjung Hospital, New Delhi,
India
${ }^{2}$ Department of Paediatrics, Vardhman Mahavir Medical College and Safdarjung hospital new Delhi, India

\author{
Correspondence: \\ Shyam Sundar Meena \\ Department of Paediatrics, \\ Vardhman Mahavir Medical College and \\ Safdarjung hospital, \\ New Delhi, India \\ Email id: dr.shyamsnmc2000@gmail.com
}

DOI: $10.3126 /$ jnps.v41i1.29595

Submitted on: 2020-09-19

Accepted on: 2021-02-15

Acknowledgements: None

Funding: Nil

Conflict of Interest: None declared

Permission from IRB: Yes

To cite this article: Meena SS. Etiological trends and epidemiological profile of tropical fever in children presenting with acute undifferentiated fever at a Tertiary Care Centre in North India. J Nepal Paediatr Soc. 2021;41(1): 54-60.

\section{ABSTRACT}

Introduction: The objective of this study was to determine the etiology of acute undifferentiated fevers (AUF) in hospitalised children with in a tertiary care centre in North India. The various causes of acute undifferentiated fevers included dengue, malaria, scrub typhus, enteric fever, leptospirosis and Chikungunya. The clinical spectrum and therapeutic outcome of these cases was also studied.

Methods: This retrospective study was conducted over a period of 6 months from June 2019 to December 2019. The clinical information of 126 children who tested positive for any of the causes of AUF namely dengue, malaria, scrub typhus, enteric fever, chikungunya and leptospirosis was obtained from the Paediatrics Department. The information was recorded in a predesigned proforma. Various tests performed for the diagnosis of these illnesses were noted. They included dengue NS1 antigen ELISA and IgM ELISA, peripheral blood smear (PBS) for malarial parasite and rapid malarial antigen detection test (RMAT), scrub typhus IgM ELISA, leptospira IgM ELISA, and Chikungunya IgM ELISA.

Results: The diagnosis of AUF were dengue (55.5\%; 70/126), malaria $(19 \% ; 24 / 126)$ and scrub typhus $(19 \% ; 24 / 126)$, enteric fever $(4.7 \% ; 6 / 126)$ and Chikungunya $(1.5 \% ; 2 / 126)$. The most common presenting symptom was fever or pyrexia followed by myalgia, generalised aches and pains in the body and vomiting. Commonly observed complications included thrombocytopenia, hepatitis and shock.

Conclusions: Dengue, malaria and scrub typhus are the major causes of AUF in the hospitalised children with dengue being the most prevalent infection. The study further emphasises the need to develop a systematic approach to the diagnosis of AUFs. This should be using a combination of clinical, epidemiological and laboratory parameters, which will be very useful for developing a relevant action plan for treatment and prevention of such fevers in any hospital setup.

Keywords: Acute undifferentiated fever (AUF); Dengue; Malaria; Scrub typhus; Tropical fevers

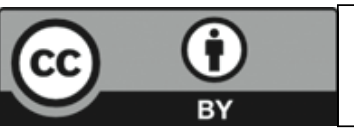

This work is licensed under creative common attribution 3.0 license 


\section{INTRODUCTION}

Infectious illnesses are the major causes of morbidity and mortality in children, particularly in the tropical countries. Acute undifferentiated fever (AUF) is a febrile illness of less than two weeks' duration, with non specific symptoms and signs such as malaise, myalgia and loss of appetite etc. AUF that are rampant in tropical and subtropical regions are collectively known as tropical fevers. ${ }^{1}$ Dengue, malaria, scrub typhus, leptospirosis, enteric fever and chikungunya are some of the common tropical fevers reported in patients from South East Asian countries, including India. Awareness and knowledge about the major febrile illnesses and their co-existence of different tropical fevers which present as AUF is of utmost importance in the treatment and management of children with these illnesses in our setup. ${ }^{2}$

Diversity exists in the trends and occurrence of these tropical fevers in hospitalised patients as they are greatly influenced by season and geography. ${ }^{3-5}$ Some of them are perennial while others demonstrate seasonal variation. These tropical fevers have overlapping clinical presentations, hence, it is imperative that the local epidemiology and etiology of these infections should be known for accurate management of these diseases. There is paucity of data on the etiology and epidemiology of these infections particularly in children. Presently available data on tropical fevers in children is very limited.

This retrospective study was planned and conducted to understand the occurrence of such infections, its epidemiology and therapeutic outcome in children attending a tertiary care teaching hospital over a period of six months.

\section{METHODS}

This retrospective study was conducted in the Paediatrics Department in collaboration with the Department of Microbiology at a tertiary care teaching hospital in North India. Ethical clearance was taken from the Institutional Ethics Committee. A waiver for getting informed consent from the patient was obtained as it was a retrospective study (IEC/VMMC/SJH/PROJECT/2019-12/88). The data of the patients (age up to 12 years) admitted in paediatric wards between June to December 2019 and presenting with undifferentiated fever of minimum five days, was collected from the patient's case records in a predesigned proforma. Those patients who had history of intake of antibiotics or anti-malarial drugs from any outside source or whose complete medical details were not available were excluded from the study. The detailed clinical examination, haematological and biochemical tests were recorded along with the treatment given and the final outcome.

Diagnosis of dengue was done by detection of NSlantigen and /or specific IgM antibodies by MAC ELISA kits supplied by National Institute of Virology (NIV), Pune, India. Testing for malarial parasite was done by examination of PBS with Giemsa stain and immuno-chromatographic test to detect lactate dehydrogenase (LDH) for Plasmodium falciparum and Plasmodium vivax and HRP2 for detection of Plasmodium falciparum (DiaSys Diagnostics Pvt Ltd, India). For diagnosis of scrub typhus, IgM ELISA (In Bios, USA), for Chikungunya, IgM ELISA (NIV, India) and for leptospirosis, leptospira IgM ELISA (Panbio Pty., Ltd., Queensland, Australia) were used as per the manufacturer's instructions. Enteric fever was diagnosed by detecting IgM antibodies using rapid immunochromatographic test (Med source ozon India) or by Widal test for detection of antibodies against the $\mathrm{O}$ and $\mathrm{H}$ antigens of $\mathrm{S}$. typhi and " $\mathrm{H}$ " antigens of S. paratyphi A. The completed data was further recorded in Microsoft Excel spreadsheets and the statistical analysis was performed using the SPSS for Windows, version 20.0 (SPSS Inc., Chicago, IL, USA).

\section{RESULTS}

A total of 4000 samples from suspected cases of AUF were screened in Microbiology Department for dengue, malaria, scrub typhus, enteric fever, leptospirosis and Chikungunya infections. Out of these, 126 samples which satisfied the selection criteria were enrolled in the study. Etiologic agents of tropical fever detected were - Dengue (70 cases), malaria (24 cases); scrub typhus (24 cases); enteric fever (Six cases); Chikungunya (Two cases). There was no case of leptospirosis. Among 126 patients, 79 were males and 47 were females. The mean age of study population was 6.94 years. Epidemiological profile and clinical features of the patients are presented in Table 1. 
Table 1. Clinical and epidemiological profile of Patients with Tropical fever

\begin{tabular}{|c|c|c|c|c|c|c|c|}
\hline Clinical feature & $\begin{array}{l}\text { Dengue } \\
(N=70)\end{array}$ & $\begin{array}{l}\text { Enteric fever } \\
\qquad(N=60)\end{array}$ & $\begin{array}{c}\text { Scrub } \\
(N=24)\end{array}$ & $\begin{array}{l}\text { Malaria } \\
(N=24)\end{array}$ & $\begin{array}{l}\text { Chikungunya } \\
\qquad(\mathrm{N}=2)\end{array}$ & $\begin{array}{c}\text { Total } \\
(\mathrm{N}=126)\end{array}$ & $\begin{array}{c}\text { Total } \\
\%\end{array}$ \\
\hline Male / Female & $40 / 30$ & $4 / 2$ & $16 / 8$ & $18 / 6$ & $1 / 1$ & $79 / 47$ & \\
\hline $\begin{array}{c}\text { Age (Years) } \\
<1 \\
1-5 \\
>5\end{array}$ & $\begin{array}{r}4 / 2 \\
10 / 9 \\
26 / 19\end{array}$ & $\begin{array}{l}0 / 0 \\
2 / 1 \\
2 / 1\end{array}$ & $\begin{array}{l}0 / 0 \\
7 / 2 \\
9 / 6\end{array}$ & $\begin{array}{r}1 / 0 \\
5 / 2 \\
12 / 4\end{array}$ & $\begin{array}{l}0 / 1 \\
0 / 0 \\
1 / 0\end{array}$ & $\begin{array}{r}5 / 3 \\
24 / 14 \\
50 / 30\end{array}$ & $\begin{array}{r}6.34 \% \\
30.15 \% \\
63.49 \%\end{array}$ \\
\hline \multicolumn{8}{|l|}{ Symptoms } \\
\hline $\begin{array}{l}\text { Fever (Days) } \\
\qquad \begin{aligned}<5 \\
5-10 \\
>10\end{aligned}\end{array}$ & $\begin{array}{r}58 \\
\\
38 \\
17 \\
3\end{array}$ & $\begin{array}{l}2 \\
2 \\
2\end{array}$ & $\begin{array}{l}2 \\
8 \\
8\end{array}$ & $\begin{array}{r}4 \\
12 \\
8\end{array}$ & $\begin{array}{l}0 \\
1 \\
1\end{array}$ & $\begin{array}{l}46 \\
40 \\
22\end{array}$ & $\begin{array}{l}36.50 \% \\
31.74 \% \\
17.46 \%\end{array}$ \\
\hline Breathing difficulty & $0(0)$ & $0(0)$ & $1(4.2)$ & $4(16.6)$ & $0(0)$ & 5 & $3.96 \%$ \\
\hline Seizure & $5(7.14)$ & $0(0)$ & $2(8.33)$ & $0(0)$ & $0(0)$ & 7 & $5.55 \%$ \\
\hline Bleeding & $6(8.6)$ & $0(0)$ & $2(8.33)$ & $2(8.33)$ & $0(0)$ & 10 & $7.93 \%$ \\
\hline Chills/rigors & $0(0)$ & $0(0)$ & $0(0)$ & $14(58.3)$ & $1(50)$ & 15 & $11.90 \%$ \\
\hline Headache & $5(7.14)$ & $1(7.2)$ & $4(16.6)$ & $6(25)$ & $0(0)$ & 16 & $12.70 \%$ \\
\hline Pain abdomen & $35(50)$ & $1(1.66)$ & $7(29.16)$ & 10 & $1(50)$ & 54 & $42.85 \%$ \\
\hline Vomiting & 42 & 3 & 8 & $12(41.6)$ & 1 & 66 & $52.38 \%$ \\
\hline Body Pain & $46(65.7)$ & $2(3.33)$ & $6(25)$ & $14(58.3)$ & $2(100)$ & 70 & $55.56 \%$ \\
\hline \multicolumn{8}{|l|}{ Signs } \\
\hline Lymphadenopathy & $0(0)$ & $0(0)$ & $0(0)$ & $0(0)$ & $0(0)$ & 0 & 0 \\
\hline Eschar & $0(0)$ & $0(0)$ & $2(8.33)$ & $0(0)$ & $0(0)$ & 2 & $1.58 \%$ \\
\hline Meningeal signs & $1(1.42)$ & $3(5)$ & $0(0)$ & $0(0)$ & $0(0)$ & 4 & $3.17 \%$ \\
\hline Jaundice & $1(1.42)$ & $0(0)$ & $2(8.33)$ & $4(16.6)$ & $0(0)$ & 7 & $5.55 \%$ \\
\hline Pericardial effusion & $6(8.57)$ & $0(0)$ & $2(8.33)$ & $0(0)$ & $0(0)$ & 8 & $6.34 \%$ \\
\hline Crackles/wheeze & $5(7.14)$ & $0(0)$ & $2(8.33)$ & $2(8.33)$ & $0(0)$ & 9 & $7.14 \%$ \\
\hline Signs of raised ICT & $7(10)$ & $1(1.66)$ & $2(8.33)$ & $1(4.16)$ & $0(0)$ & 11 & $8.73 \%$ \\
\hline Edema & $8(11.4)$ & $2(3.33)$ & $3(12.5)$ & $0(0)$ & $0(0)$ & 13 & $10.31 \%$ \\
\hline Pleural effusion & $10(14.2)$ & $0(0)$ & $2(8.33)$ & $1(4.16)$ & $0(0)$ & 13 & $10.31 \%$ \\
\hline Ascites & $11(15.7)$ & $1(1.66)$ & $1(4.16)$ & $1(4.16)$ & $0(0)$ & 14 & $11.12 \%$ \\
\hline Splenomegaly & $1(1.42)$ & $2(3.33)$ & $2(8.33)$ & $10(41.6)$ & $0(0)$ & 15 & $11.90 \%$ \\
\hline Rash & $15(21.42)$ & $1(1.66)$ & $3(12.5)$ & $0(0)$ & $2(100)$ & 21 & $16.66 \%$ \\
\hline Pallor & $4(5.71)$ & $3(5)$ & $4(16.6)$ & $9(37.5)$ & $0(0)$ & 20 & $15.87 \%$ \\
\hline $\begin{array}{l}\text { Tachypnea/ chest } \\
\text { retraction }\end{array}$ & $8(11.4)$ & $2(3.33)$ & $6(25)$ & $4(16.6)$ & $0(0)$ & 20 & $15.87 \%$ \\
\hline Altered sensorium & $14(20)$ & $3(5)$ & $8(33.3)$ & $4(16.6)$ & $0(0)$ & 29 & $23.01 \%$ \\
\hline Evidence of shock & $21(30)$ & $3(5)$ & $6(25)$ & $7(29.1)$ & $0(0)$ & 37 & $29.36 \%$ \\
\hline Hepatomegaly & $20(28.57)$ & $5(8.33)$ & $14(58.3)$ & $13(54.2)$ & $0(0)$ & 52 & $41.26 \%$ \\
\hline
\end{tabular}

The observed haematological alterations were thrombocytopenia in 74 cases. Thrombocytopenia was observed in $79 \%(19 / 24)$ of the patients with scrub typhus, $62 \%(44 / 70)$ of the dengue cases and $45 \%(11 / 24)$ of patients with malaria. Pancytopenia was seen in $8 / 126$ cases $(6.4 \%)$. Out of these, four were positive for dengue, three for scrub typhus and one for malaria.

Biochemical, haematological and other laboratory parameters of all the cases have been shown in Table 2. 
Table 2. Laboratory investigations of patients with Tropical fevers

\begin{tabular}{|c|c|c|c|}
\hline \multicolumn{2}{|c|}{ Biochemical Parameters } & $\begin{array}{c}\text { Total } \\
(\mathrm{N}=126)\end{array}$ & $\begin{array}{c}\text { Total } \\
\text { Percentage } \\
(\%)\end{array}$ \\
\hline \multirow{3}{*}{$\begin{array}{l}\text { Aspartate } \\
\text { amino- } \\
\text { transferase } \\
(\text { AST })>40 \\
\text { IU/l }\end{array}$} & $<2 \mathrm{x}$ & 26 & 20.63 \\
\hline & $2 x-4 x$ & 23 & 18.25 \\
\hline & $>4 \mathrm{x}$ & 35 & 27.7 \\
\hline \multirow{3}{*}{$\begin{array}{l}\text { Alanine } \\
\text { transaminase } \\
(\mathrm{ALT})>\mathbf{4 0} \\
\text { IU/l }\end{array}$} & $<2 \mathrm{x}$ & 19 & 15.07 \\
\hline & $2 x-4 x$ & 10 & 7.93 \\
\hline & $>4 x$ & 29 & 23.01 \\
\hline \multicolumn{2}{|l|}{$\begin{array}{l}\text { Bilirubin } \\
(>1.2 \mathrm{mg} \%)\end{array}$} & 23 & 18.25 \\
\hline \multicolumn{2}{|l|}{$\begin{array}{l}\text { Altered } \\
\text { coagulation } \\
\text { profile (PT/ } \\
\text { INR) }\end{array}$} & 33 & 26.19 \\
\hline \multicolumn{2}{|l|}{$\begin{array}{l}\text { Raised } \\
\text { Creatinine } \\
(>1 \mathrm{mg} / \mathrm{dl})\end{array}$} & 8 & 6.34 \\
\hline \multicolumn{2}{|c|}{ Hematologic parameters } & $\begin{array}{c}\text { Total } \\
(\mathrm{N}=126)\end{array}$ & $\begin{array}{c}\text { Total } \\
\text { Percentage } \\
(\%)\end{array}$ \\
\hline \multirow{3}{*}{$\begin{array}{l}\text { Hemoglobin } \\
\text { (gm \%) }\end{array}$} & 8 to 10 & 45 & 35.71 \\
\hline & 6 to 8 & 21 & 16.6 \\
\hline & $<6$ & 3 & 2.8 \\
\hline \multirow{3}{*}{$\begin{array}{l}\text { Total } \\
\text { leucocyte } \\
\text { count } \\
\text { (per cu.mm) }\end{array}$} & $<4000$ & 10 & 7.94 \\
\hline & $4000-11000$ & 76 & 60.32 \\
\hline & $>11000$ & 40 & 31.74 \\
\hline \multirow{4}{*}{$\begin{array}{l}\text { Platelets } \\
\text { (per cu.mm) }\end{array}$} & $100000-150000$ & 11 & 8.73 \\
\hline & $50000-100000$ & 28 & 22.22 \\
\hline & $20000-50000$ & 23 & 18.25 \\
\hline & $<20000$ & 12 & 9.52 \\
\hline \multicolumn{2}{|l|}{ Pancytopenia } & 8 & 6.34 \\
\hline
\end{tabular}

Etiologic agents detected were dengue (55.5\%; $70 / 126)$, malaria $(19 \% ; 24 / 126)$; scrub typhus $(19 \% ; 24 / 126)$, enteric fever $(4.7 \% ; 6 / 126)$ and chikungunya $(1.5 \% ; 2 / 126)$. Co-infections of malaria and enteric fever were recorded in eight, dengue and malaria in five, dengue and enteric fever in three, malaria and scrub typhus in two and enteric fever and scrub typhus in two patients. Not a single case of leptospirosis was reported in our study. Out of 126 patients, 118 were successfully treated and discharged, and eight succumbed. All eight patients who died were dengue positive.
Table 3. Complications observed in patients with Tropical fever

\begin{tabular}{|lr|}
\hline Complication & No of cases (\%) \\
\hline Hepatitis & $58(46.03 \%)$ \\
Shock & $28(22.22 \%)$ \\
CHF & $11(8.73 \%)$ \\
Signs of raised ICT & $11(8.73 \%)$ \\
HLH & $10(7.93 \%)$ \\
DIC & $10(7.93 \%)$ \\
Respiratory failure & $10(7.93 \%)$ \\
Meningoencephalitis & $6(4.76 \%)$ \\
Acute renal failure & $5(3.96 \%)$ \\
Pneumonia & $5(3.96 \%)$ \\
ARDS & $4(3.17 \%)$ \\
Cardiac dysfunction & $2(1.58 \%)$ \\
\hline
\end{tabular}

Lumbar puncture was done in 26 patients who showed signs of raised ICT or meningitis. Abnormal CSF findings (raised proteins and decreased sugar ) were obtained in six patients. All CSF cultures were sterile. Hepatitis, shock, haemophagocytic lymphohistocytosis (HLH), DIC, ARDS, CHF and raised ICT were some common complications observed in the present study. HLH, an atypical complication was observed in 10 cases (Four dengue, three scrub typhus, two malaria and one enteric fever). Various other complications found have been depicted in Table 3.

\section{DISCUSSION}

Tropical fevers pose a major problem in the tropical regions of the world. India constitutes a major chunk of tropical infections, which are either vector borne or food borne. These tropical fevers often present as AUF with overlapping symptoms and signs and hence making it difficult to diagnose clinically.

In our study, amongst the paediatric patients with acute febrile illness and its systemic manifestations, dengue, malaria and scrub typhus were found to be the commonest etiologies followed by enteric fever and Chikungunya. These results are comparable to other studies from India and other South East Asian countries with slight variable results. ${ }^{6-10}$ Such 
variation may have resulted because of various geographical status and local endemicity. A high number of dengue cases in our study could be attributed to the endemicity of dengue in our region.

In regards to clinical presentation, fever was the most common presenting feature, followed by myalgia / generalised body pain, vomiting and rashes. We noted that fever was the presenting feature in all patients with malaria and enteric fever. Fever was present in 58/70 (82.9\%) cases of dengue and 18/24 (75\%) cases of scrub typhus. This implies that some of the cases of dengue and scrub typhus may have been afebrile at the time of presentation. Similarly chills and rigors were also observed in 15/24 (62.5\%) cases of malaria.

Rash was the predominant sign of dengue $15 / 70$ (21.4\%) and chikungunya (2/2) patients. Eschar, which is a pathognomonic sign of scrub typhus was seen only in $2 / 24(8.3 \%)$ cases, which is much less than that published by other workers from our region. ${ }^{11}$ Although bleeding manifestations were observed in more number of cases of dengue $6 / 70$ $(8.6 \%)$, the percentage of cases presenting with bleeding manifestations remained same for both scrub typhus (8.33\%) and malaria (8.33\%).

There are published case reports / series where the CNS involvement presenting either as meningitis and / or encephalitis has been seen in patients with dengue and scrub typhus. ${ }^{12-14}$ We found CNS manifestations in $50 \%$ scrub typhus cases and $38.6 \%$ of dengue cases. CNS manifestations that were observed were seizures, altered sensorium, raised intra cranial pressure and meningeal signs.

We found thrombocytopenia in $79 \%$ of patients with scrub typhus, $62 \%$ of dengue cases and $45 \%$ of patients with malaria. Pancytopenia was seen with remarkable predominance in dengue and scrub typhus as compared to malaria. The possible underlying causes of pancytopenia in tropical fevers are bone marrow hypoplasia, haemophagocytic syndrome or secondary to microangiopathic haemolytic anaemia. Haemophagocytic syndrome (HPS) is one of the key players in the pathogenesis of pancytopenia in various tropical fevers. ${ }^{15}$
HLH or HPS is suspected in patients presenting with high-grade fever, splenomegaly, pancytopenia and multi-system involvement. The findings of raised serum lactate dehydrogenase $(\mathrm{LDH})$, ferritin and triglyceride and / or a low fibrinogen further increases the probability of HLH. Karnad et al. found an association of HLH with the tropical fevers like dengue, malaria, tuberculosis, hepatitis A, enteric fever, rickettsia, leptospirosis, chikungunya, meningococcemia, HIV, infectious mononucleosis etc. ${ }^{15}$ In our study HLH was seen in almost all reported tropical fevers except in chikungunya. These findings suggest that HLH may complicate any of the cases presenting with AUF.

Some studies have tried to examine the various clinical and laboratory parameters to differentiate between tropical fevers, while others have tried to develop a scoring system to differentiate between the various causes of undifferentiated fevers. ${ }^{8,16}$ Some studies have reported leukocytosis, elevated alanine aminotransferase (ALT), elevated aspartate aminotransferase (AST), low serum albumin as significant predictors of scrub typhus when compared to other AUF. ${ }^{8,16}$ Similar findings were observed in our study also. In our study leucocytosis was observed in $33 \%$, while elevated AST and ALT were observed in $75 \%$ and $67 \%$ of the patients with scrub typhus respectively. Such phenomenon in scrub typhus can be attributed to intrahepatic sinusoidal endothelial vasculitis caused by Orientia tsutsugamushi. ${ }^{17,18}$ Varying degrees of liver involvement in tropical fevers may be explained from hepatocyte apoptosis caused directly by the infective agent, impaired liver perfusion resulting from fluid leakage, oxidative stress or immune mediated injury.

In our study, the common complications were hepatitis, shock, CHF, raised ICT, DIC along with some atypical complications like HLH, meningoencephalitis, renal failure and ARDS. Besides various complications, there were total eight patients who had died. We also noted that all the mortality in our study were due to dengue. Dengue is known to be a relatively more fatal disease among AUF, and the higher prevalence of dengue in our study may have led to such finding. 
This retrospective study had certain limitations. As this study is a single centric study, there can be a patient population bias in the patient selection and their referral pattern. Gold standard tests for these various AUF could not be performed for confirmation due to limited resources. Since our hospital is a tertiary care centre, the referral bias may be far higher for the reported complication rates.

\section{CONCLUSIONS}

Dengue, malaria, scrub typhus, enteric fever etc are the common tropical fevers found in the children in our region. This retrospective study highlights the emerging problem of scrub typhus in our setup. Details of the various biochemical and haematological parameters along with atypical manifestations like HLH observed in the children in the present study will greatly assist the clinicians by increasing their awareness of the varying manifestations of these tropical fevers. This study and similar data on AUF in the future should be very useful in developing a relevant action plan to address this public health issue.

\section{REFERENCES}

1. Singhi S, Chaudhary D, Varghese GM, Bhalla A, Karthi N, Kalantri S, et al. Tropical fevers: Management guidelines. Indian J Crit Care Med. 2014 Feb;18(2):62-9. DOI: 10.4103/0972-5229.126074.

2. Arora BS, Matlani M, Saigal K, Biswal I, Rajan S, Padmanandan A, et al. Major aetiologies of acute undifferentiated fever in 2013 and 2014: an experience in retrospect. Int J Adv Med. 2017 Apr;4(2):568-72. DOI: http://dx.doi.org/10.18203/2349-3933.ijam20171062

3. Leelarasamee A, Chupaprawan C, Chenchittikul M, Udompanthurat S. Etiologies of acute undifferentiated febrile illness in Thailand. J Med Assoc Thai. 2004 May;87(5):464-72. PMID: 15222513.

4. Wangdi K, Kasturiaratchi K, Nery SV, Lau CL, Gray D J, Clements ACA. Diversity of infectious aetiologies of acute undifferentiated febrile illnesses in south and Southeast Asia: a systematic review. BMC Infect Dis. 2019;19:577. DOI: https://doi.org/10.1186/s12879-019-4185-y.

5. Wangrangsimakul T, Althaus T, Mukaka M, Kantipong P, Wuthiekanun P, Wirongrong C, et al. Causes of acute undifferentiated fever and the utility of biomarkers in Chiangrai, Northern Thailand. PLoS Negl Trop Dis. 2018;12:e0006477. DOI: 10.1371/journal.pntd.0006477

6. Raina S, Raina RK, Agarwala N, Raina SK, Sharma R. Coinfections as an aetiology of acute undifferentied febrile illness among adult patients in the sub-Himalayan region of north India. J Vector Borne Dis. 2018;55(2):130-6. DOI: : 10.4103/0972-9062.242560

7. Singhi S, Rungta N, Nallasamy K, Bhall A, Peter JV, Chaudhry D et al. Tropical Fevers in Indian Intensive Care Units: A Prospective Multicenter Study. Indian J Crit Care Med. 2017;21(12):811-8. DOI:10.4103/ ijccm.IJCCM_324_17

8. Abrahamsen SK, Haugen CN, Rupali P, Mathai D, Langeland N, Eide GE, et al. Fever in the tropics: Aetiology and case-fatality - A prospective observational study in a tertiary care hospital in South India. BMC Infect Dis. 2013;13:355. DOI: 10.1186/1471-2334-13-355

9. Chrispal A, Boorugu H, Gopinath KG, Chandy S, Prakash JA, Thomas EM, et al. Acute undifferentiated febrile illness in adult hospitalized patients: The disease spectrum and diagnostic predictors - An experience from a tertiary care hospital in South India. Trop Doct. 2010;40(4):230-34. DOI: https://doi.org/10.1258/td.2010.100132

10. Ellis RD, Fukuda MM, McDaniel P, Welch K, Nisalak A, Murray CK, et al. Causes of fever in adults on the ThaiMyanmar border. Am J Trop Med Hyg. 2006;74:108-13. DOI: 10.4269/ajtmh.2006.74.108

11. Mina SS, Kumar V, Chhapola V. Emerging Infections in Children in North India: Scrub Typhus. J Pediatr Infect Dis. 2017;12:114-8. DOI: 10.1055/s-0037-1599835 
12. Sarkar M, Datta D, Biswas T, Roychowdhoury S. Acute Encephalitis Syndrome Due to Scrub Typhus Infection in Pediatric Population: A Case Series from a Tertiary Care Center of Eastern India. J Pediatr Infect Dis. 2016;11(02): 37-41.DOI: 10.1055/s-0036-1587599

13. Madi D, Achappa B, Ramapuram JT, Chowta N, Laxman M, Mahalingam S. Dengue Encephalitis - A Rare Manifestation of Dengue Fever. Asian Pac J of Trop Biomed. 2014 May:4(1):70-2. DOI: 10.12980/APJTB. $4.2014 \mathrm{C} 1006$

14. Watt G, Jongsakul K, Chouriyagune C, Paris R. Differentiating dengue virus infection from scrub typhus in Thai adults with fever. Am J Trop Med Hyg. 2003;68:536-8. DOI: https://doi.org/10.4269/ajtmh.2003.68.536

15. Karnad DR, Richards GA, Silva GS, Amin P. HLH like disease vs. true HLH in tropical disease. J Crit Care. 2018 Dec;48:469-70. DOI: 10.1016/j.jcrc.2018.09.019.

16. Kodan P, Chakrapani M, Shetty M, Pavan R, Bhat P. Hemophagocytic lymphohistiocytosis secondary to infections: a tropical experience. J Postgrad Med. 2015;61(2):112-5. DOI: 10.4103/0022-3859.150904

17. Mitra S, Gautam I, Jambugulam M, Abhilash KP, Jayaseeelan V. Clinical Score to Differentiate Scrub Typhus and Dengue: A Tool to Differentiate Scrub Typhus and Dengue. J Glob Infect Dis. 2017;9(1):12-7. DOI: 10.4103/0974-777X.199996

18. Chung JH, Lim SC, Yun NR, Shin SH, Kim CM, Kim DM. Scrub typhus hepatitis confirmed by immunohistochemical staining. World J Gastroenterol. 2012;18(36):5138-41. DOI:10.3748/wjg. v18.i36.5138 